\title{
A uniform result for the dimension of fractional Brownian motion level sets
}

\section{Lara Daw*}

Université Paris-Est, LAMA(UMR8050), UPEMLV, UPEC, CNRS, F-94010 Cr éteil, France

University of Luxembourg, UR en Mathématiques, Maison du nombre, 6 avenue de la Fonte, L-4364 Esch-sur-Alzette, Luxembourg

\section{A R T I C L E I N F O}

\section{Article history:}

Received 3 March 2020

Received in revised form 12 October 2020

Accepted 17 October 2020

Available online 6 November 2020

\section{Keywords:}

Level sets

Fractional Brownian motion

Local times

Macroscopic Hausdorff dimension

\begin{abstract}
A B S T R A C T
Let $B=\left\{B_{t}: t \geq 0\right\}$ be a real-valued fractional Brownian motion of index $H \in(0,1)$. We prove that the macroscopic Hausdorff dimension of the level sets $\mathcal{L}_{x}=\left\{t \in \mathbb{R}_{+}: B_{t}=x\right\}$ is, with probability one, equal to $1-H$ for all $x \in \mathbb{R}$.
\end{abstract}

(C) 2020 Elsevier B.V. All rights reserved.

\section{Introduction}

Let $B=\left\{B_{t}: t \geq 0\right\}$ be a fractional Brownian motion of index $H \in(0,1)$, that is, a centered, real-valued Gaussian process with covariance function

$$
R(s, t)=\mathbb{E}\left(B_{s} B_{t}\right)=\frac{1}{2}\left(|s|^{2 H}+|t|^{2 H}-|s-t|^{2 H}\right), \quad s, t \geq 0 .
$$

Since $\mathbb{E}\left[\left(B_{s}-B_{t}\right)^{2}\right]=|s-t|^{2 H}$, it is an immediate consequence of the Kolmogorov-Chentsov continuity theorem that $B$ admits a continuous modification. Throughout this note, we will always assume that $B$ is continuous. It is also immediate (see, e.g., Nourdin, 2012) that $B$ is a self-similar process of exponent $H$, that is, for any $a>0$,

$$
\left\{B_{a t}: t \geq 0\right\} \stackrel{d}{=}\left\{a^{H} B_{t}: t \geq 0\right\},
$$

where $X \stackrel{d}{=} Y$ means that two processes $X$ and $Y$ have the same distribution. Moreover, $B$ has stationary increments, that is, for every $s \geq 0$,

$$
\left\{B_{t+s}-B_{s}: t \geq 0\right\} \stackrel{d}{=}\left\{B_{t}: t \geq 0\right\} .
$$

This article is concerned with estimating the size of the level sets of $B$, which are defined for any $x \in \mathbb{R}$ as

$$
\mathcal{L}_{x}=\left\{t \geq 0: B_{t}=x\right\} .
$$

\footnotetext{
* Correspondence to: University of Luxembourg, UR en Mathématiques, Maison du nombre, 6 avenue de la Fonte, L-4364 PEsch-surAlzette, Luxembourg.

E-mail address: lara.daw@uni.lu.
} 
This line of research started with the seminal work of Taylor (1955), who was the first to study the Hausdorff dimensions of the level sets in the case of a standard Brownian motion. His results were extended later on by Perkins (1981) who showed that, with probability one, the level sets $\mathcal{L}_{x}$ have a Hausdorff dimension $\frac{1}{2}$ for all $x \in \mathbb{R}$. Hence, the local structure of the level sets in the Brownian case is well understood.

Another method to describe the geometric properties of the sample paths of a given process is in terms of its sojourn times. Here, the goal is to study the dimension of the amount of time spent by the stochastic process inside a moving boundary, that is, of the form

$$
E(\phi):=\left\{t \geq 0:\left|B_{t}\right| \leq \phi(t)\right\}
$$

where $\phi: \mathbb{R}_{+} \rightarrow \mathbb{R}$ is an appropriate function.

Strongly related to our note, we mention the recent work of Nourdin et al. (2018), in which a specific large scale dimension is computed for the sojourn times

$$
E_{\gamma}:=\left\{t \geq 0:\left|B_{t}\right| \leq t^{\gamma}\right\}, \quad 0<\gamma<H,
$$

of the fractional Brownian motion $B$. Note that this choice for $\phi$ is completely natural here because, on the one hand, the fractional Brownian motion is selfsimilar (hence the choice of a power function for $\phi$ ) and, on the other hand, it satisfies a law of iterated logarithm as $t \rightarrow \infty$ (hence the range $(0, H)$ for $\gamma$ ). Actually, Nourdin et al. (2018) extended to the fractional Brownian motion the results given by Seuret and Yang (2019) in the framework of the standard Brownian case.

In general, defining a notion of fractal dimension for a subset of $\mathbb{R}^{d}$ involves taking into consideration the microscopic (i.e. local) properties of this set. However, many models in statistical physics are based on the Euclidean lattice $\mathbb{Z}^{d}$; in this case, it may look more natural to rely on the macroscopic (i.e. global) properties of the set to define a notion of dimension. This is what Barlow and Taylor proposed in Barlow and Taylor $(1989,1992)$. Their dimension, called macroscopic Hausdorff dimension, has proven to be relevant in many contexts. This is the one that was used in Nourdin et al. (2018) and Seuret and Yang (2019), and also the one we will use in the present note, because it can give a good intuition about the geometry of the set into consideration, precisely whether it is scattered or not. Precise definitions will be given in Section 2.1. At this stage, we only mention that we denote this macroscopic Hausdorff dimension by $\operatorname{Dim}_{H}$.

Our note can be considered as an addendum to Nourdin et al. (2018). Let $\mathcal{L}_{\chi}$ be the level sets associated with a fractional Brownian motion. In Nourdin et al. (2018), the following is shown.

Theorem 1. Fix $x \in \mathbb{R}$. Then

$$
\mathbb{P}\left(\operatorname{Dim}_{H} \mathcal{L}_{x}=1-H\right)=1 .
$$

Our aim is to extend Theorem 1 from " $\forall x, \mathbb{P}(\ldots)=1$ " to " $\mathbb{P}(\forall x: \ldots)=1$ ". To this end, new and non-trivial arguments are required. We will prove the following.

\section{Theorem 2.}

$$
\mathbb{P}\left(\forall x \in \mathbb{R}: \operatorname{Dim}_{H} \mathcal{L}_{x}=1-H\right)=1 .
$$

We note that our Theorem 2 also recovers Seuret-Yang's result (Seuret and Yang, 2019, Theorem 2) (Brownian motion), and provides a proof that we find more natural.

Throughout the note, every random object is defined on a common probability space $(\Omega, \mathcal{A}, \mathbb{P})$, and $\mathbb{E}$ denotes the expectation with respect to $\mathbb{P}$.

\section{Preliminaries}

This section gathers the different tools that will be needed in order to prove Theorem 2 .

\subsection{Macroscopic Hausdorff dimension}

Following the notations of Khoshnevisan et al. (2017) and Khoshnevisan and Xiao (2017), we consider the intervals $S_{-1}=[0,1 / 2)$ and $S_{n}=\left[2^{n-1}, 2^{n}\right)$ for $n \in \mathbb{N}$. For $E \subset \mathbb{R}^{+}$, we define the set of proper covers of $E$ restricted to $S_{n}$ by

$$
\mathcal{I}_{n}(E)=\left\{\begin{array}{rc}
\left\{I_{i}\right\}_{i=1}^{m}: & I_{i}=\left[x_{i}, y_{i}\right] \text { with } x_{i}, y_{i} \in \mathbb{N}, y_{i}>x_{i} \\
& I_{i} \subset S_{n} \text { and } E \cap S_{n} \subset \bigcup_{i=1}^{m} I_{i} .
\end{array}\right\}
$$

For any set $E \subset \mathbb{R}^{+}, \rho \geq 0$ and $n \geq-1$, we define

$$
v_{\rho}^{n}(E)=\inf \left\{\sum_{i=1}^{m}\left(\frac{\operatorname{diam}\left(I_{i}\right)}{2^{n}}\right)^{\rho}:\left\{I_{i}\right\}_{i=1}^{m} \in \mathcal{I}_{n}(E)\right\},
$$

where $\operatorname{diam}([a, b])=b-a$.

The key point in the definition of $v_{\rho}^{n}(E)$ is that the sets $I_{i}$ are non-trivial intervals with integer boundaries; in particular, the infimum is reached. 
Definition 3. Let $E \subset \mathbb{R}^{+}$. The macroscopic Hausdorff dimension of $E$ is defined by

$$
\operatorname{Dim}_{H} E=\inf \left\{\rho>0: \sum_{n \geq-1} v_{\rho}^{n}(E)<+\infty\right\} .
$$

We observe that $\operatorname{Dim}_{H} E$ always belongs to $[0,1]$, whatever $E \subset \mathbb{R}^{+}$. Indeed, consider the family $I_{i}=\left[2^{n-1}+i-\right.$ $\left.1,2^{n-1}+i\right], 1 \leq i \leq 2^{n-1}$, which belongs to $\mathcal{I}_{n}(E)$ and satisfies $\sum_{i=1}^{m}\left(\frac{\operatorname{diam}\left(I_{i}\right)}{2^{n}}\right)^{\rho} \leq \frac{1}{2} 2^{n(1-\rho)}$. Thus, $v_{1+\varepsilon}^{n}(E) \leq 2^{-n \varepsilon}$ for all $\varepsilon>0$, implying in turn that $\operatorname{Dim}_{H} E \leq 1+\varepsilon$ for all $\varepsilon>0$. As a result, we have that $\operatorname{Dim}_{H} E \in[0,1]$.

In (5), the covers are chosen to have length larger than 1 . This shows that the macroscopic Hausdorff dimension does not rely on the local structure of the underlying set.

The dimension of a set is unchanged when one removes any bounded subset, since the series in (6) converges if and only if its tail series converges. Consequently, the dimension of any bounded set $E$ is zero. But the converse is not true, for example $\operatorname{Dim}_{H}\left(\left\{2^{n}, n \geq 1\right\}\right)=0$.

The macroscopic Hausdorff dimension not only counts the number of covers of a set but also it gives an intuition about the geometry of the set. Precisely, the more the points of the set are spread-out, the larger its dimension. For instance for $0<\alpha<1$, define the two sets $A_{\alpha}$ and $B_{\alpha}$ by for all $n \geq 1$,

$$
\begin{aligned}
& A_{\alpha} \cap S_{n}=\left\{2^{n-1}+k \frac{2^{n-1}}{2^{n \alpha}}: k \in\left\{0, \ldots, 2^{n \alpha}-1\right\}\right\} ; \\
& B_{\alpha} \cap S_{n}=\left\{2^{n-1}+\frac{k}{2^{n \alpha}}: k \in\left\{0, \ldots, 2^{n \alpha}-1\right\}\right\} .
\end{aligned}
$$

Even though both sets have same cardinality but $\operatorname{Dim}_{H} A_{\alpha}=\alpha$ whereas $\operatorname{Dim}_{H} B_{\alpha}=0$.

These features make the macroscopic Hausdorff dimension an interesting quantity describing the large scale geometry of a set; in particular, it appears to be well suited for the study of the level sets $\mathcal{L}_{x}$.

As we will see in our upcoming analysis, it might be sometimes wise to slightly modify the way $\operatorname{Dim}_{H} E$ is defined, to get a definition that is more amenable to analysis. For this reason, let us introduce, for any $E \subset \mathbb{R}^{+}, \rho>0, \xi \geq 0$, and $n \geq-1$, the quantity

$$
\widetilde{v}_{\rho, \xi}^{n}(E)=\inf \left\{\sum_{i=1}^{m}\left(\frac{\operatorname{diam}\left(I_{i}\right)}{2^{n}}\right)^{\rho}\left|\log _{2} \frac{\operatorname{diam}\left(I_{i}\right)}{2^{n}}\right|^{\xi}:\left\{I_{i}\right\}_{i=1}^{m} \in \mathcal{I}_{n}(E)\right\} .
$$

The difference between $v_{\rho}^{n}(E)$ and $\widetilde{v}_{\rho, \xi}^{n}(E)$ is that we introduce a logarithmic factor in the latter. This modification has actually no impact on the definition of $\operatorname{Dim}_{H} E$, as stated by the following lemma.

Lemma 4. Let $\xi \geq 0$. For every set $E \subset \mathbb{R}^{+}$,

$$
\operatorname{Dim}_{H} E=\inf \left\{\rho>0: \sum_{n \geq-1} \widetilde{v}_{\rho, \xi}^{n}(E)<+\infty\right\} .
$$

Proof. Define $\tilde{d}_{\xi}=\inf \left\{\rho>0: \sum_{n \geq-1} \widetilde{v}_{\rho}^{n, \xi}(E)<+\infty\right\}$. For $n \geq-1$, consider $\left\{I_{i}\right\}_{i=1}^{m} \in \mathcal{I}_{n}(E)$. As $I_{i} \subset S_{n}$, one has $\operatorname{diam}\left(I_{i}\right) \leq 2^{n-1}$, implying in turn that $\left|\log _{2} \frac{\operatorname{diam}\left(I_{i}\right)}{2^{n}}\right|^{\xi} \geq 1$. Thus, $\widetilde{v}_{\rho, \xi}^{n}(E) \geq v_{\rho}^{n}(E)$ and then $\operatorname{Dim}_{H} E \leq \tilde{d}_{\xi}$.

If $\operatorname{Dim}_{H} E=1$, the conclusion is straightforward. So, let us assume that $\operatorname{Dim}_{H} E<1$ and let us fix $\epsilon>0$ small enough and $\rho<1$ such that $\rho>\operatorname{Dim}_{H} E+\epsilon$. Since the function $x \mapsto x^{\epsilon}\left|\log _{2} x\right|^{\xi}$ is continuous on $(0,1]$ and tends to zero as $x$ tends to zero, it follows that there exists $c>0$ such that

$$
\left|\log _{2} x\right|^{\xi} \leq c x^{-\epsilon}, \forall x \in(0,1]
$$

We deduce that, for all $\left\{I_{i}\right\}_{i=1}^{m} \in \mathcal{I}_{n}(E)$,

$$
\sum_{i=1}^{m}\left(\frac{\operatorname{diam}\left(I_{i}\right)}{2^{n}}\right)^{\rho}\left|\log _{2} \frac{\operatorname{diam}\left(I_{i}\right)}{2^{n}}\right|^{\xi} \leq c \sum_{i=1}^{m}\left(\frac{\operatorname{diam}\left(I_{i}\right)}{2^{n}}\right)^{\rho-\epsilon}
$$

By taking the infimum over all $\left\{I_{i}\right\}_{i=1}^{m} \in \mathcal{I}_{n}(E)$ and recalling the definitions (5) and (7), one deduces that $\widetilde{v}_{\rho, \xi}^{n}(E) \leq c v_{\rho-\epsilon}^{n}(E)$, implying in turn $\widetilde{d}_{\xi} \leq \rho-\epsilon$. Letting $\rho$ tend to $\operatorname{Dim}_{H} E+\epsilon$ yields the result.

\subsection{Local time of fractional Brownian motion}

As we will see, the use of the local time will play a key role throughout the proof of Theorem 2. 
Provided it exists, the local time $x \mapsto L_{t}^{x}$ of a given process $\left(X_{t}\right)_{t>0}$ is, for each $t$, the density of the occupation measure $\mu_{t}(A)=\lambda\left(\left\{s \in[0, t]: X_{s} \in A\right\}\right)$ associated with $X$, where $\lambda$ stands for the Lebesgue measure; otherwise stated, one has $L_{t}=\frac{d \mu_{t}}{d \lambda}$. In what follows, we shall also freely use the notation $L_{t}([a, b])$ to indicate the quantity $L_{t}(b)-L_{t}(a)$.

The case where $X$ is Gaussian (and centered, say) has been widely studied in the literature. For instance, we can refer to the survey by Dozzi (2003). One of the main striking results in the Gaussian framework is the following easy-to-check condition that ensures that $\left(L_{t}^{X}\right)_{t \in[0, T], x \in \mathbb{R}}$ exists in $L^{2}(\Omega)$ :

$$
I:=\iint_{[0, T]^{2}} \frac{d s d t}{\sqrt{R(s, s) R(t, t)-R(s, t)^{2}}}<+\infty
$$

where $R(s, t)=\mathbb{E}\left(X_{s} X_{t}\right)$; moreover, in this case we have the Fourier type representation:

$$
L_{t}^{x}=\frac{1}{2 \pi} \int_{\mathbb{R}} d y \int_{0}^{t} d u e^{i y\left(B_{u}-x\right)} .
$$

If $X$ is Gaussian, selfsimilar of index $H$ and satisfies (9), then it is immediate from (10) that its local time at level $x$ also have some selfsimilarity properties in time with index $1-H$, but with a different level as stated below. More precisely, one has, for every $c>0$ :

$$
\left(L_{c t}^{x}\right)_{t \geq 0, x \in \mathbb{R}} \stackrel{d}{=} c^{1-H}\left(L_{t}^{c^{-H} x}\right)_{t \geq 0, x \in \mathbb{R}} .
$$

When $X$ stands for the fractional Brownian motion $B$ of Hurst index $H \in(0,1)$, it is immediate that (9) and (11) are satisfied. But we can go further. A consequence of Berman's work (Berman and Getoor, 1973) is that the local time associated to $B$ is $\beta$-Hölder continuous in $t$ for every $\beta \leq 1-H$ and uniformly in $x$. On their side, German and Horowitz (see Geman and Horowitz, 1980, Theorem 26.1) proved that, for all fixed $t$, the local time $\left(L_{t}^{x}\right)_{x \in \mathbb{R}}$ admits the Hölder regularity in space stated in the following lemma.

Lemma 5 (Spatial Hölder Continuity of Local Time). Assume $X$ is a fractional Brownian motion of Hurst index $H \in(0,1)$ and consider its local time $\left(L_{t}^{X}\right)_{x \in K}$, where $K$ is a given compact interval in $\mathbb{R}$. Then, for all $\beta \in\left(0, \frac{1}{2}\left(\frac{1}{H}-1\right)\right)$ and for all $t \geq 0$,

$$
\mathbb{P}\left(\sup _{x, y \in K} \frac{\left|L_{t}^{x}-L_{t}^{y}\right|}{|x-y|^{\beta}}<\infty\right)=1 .
$$

As we will see, Lemma 5 will be one of our main key tools in order to prove Lemma 8 (which is one of the steps leading to the proof of Theorem 2).

\subsection{Filtration of fractional Brownian motion}

A last crucial property of the fractional Brownian $B$ that we will use in order to prove Theorem 2, is that the natural filtration associated with $B$ is Brownian. We mean by this that there exists a standard Brownian motion $\left(W_{u}\right)_{u \geq 0}$ defined on the same probability space than $B$ such that its filtration satisfies, for all $t>0$,

$$
\sigma\left\{B_{u}: u \leq t\right\} \subset \sigma\left\{W_{u}: u \leq t\right\} .
$$

Property (13) is an immediate consequence of the Volterra representation of $B$ (see, e.g., Baudoin and Nualart, 2003). It will be exploited together with the Blumenthal's 0-1 law, in the end of the proof of Proposition 6.

\section{Proof of Theorem 2}

\subsection{Upper bound for $\operatorname{Dim}_{H} \mathcal{L}_{x}$}

By a theorem in Nourdin et al. (2018), for every $\gamma \in(0, H)$, a.s.

$$
\operatorname{Dim}_{H} E_{\gamma}=1-H \text {. }
$$

On the other hand, observe that for a fixed $\gamma>0$ and $x \in \mathbb{R}$, the level set $\mathcal{L}_{x}$ is ultimately included in $E_{\gamma}$. Indeed,

$$
\mathcal{L}_{x} \cap\left[|x|^{1 / \gamma},+\infty\right) \subset E_{\gamma} .
$$

We have recalled in Section 2.1 that the macroscopic Hausdorff dimension is insensitive to the suppression of any bounded subset. As a result, a.s. for every $x \in \mathbb{R}$,

$$
\operatorname{Dim}_{H} \mathcal{L}_{x}=\operatorname{Dim}_{H}\left(\mathcal{L}_{x} \cap\left[|x|^{1 / \gamma},+\infty\right)\right) \leq \operatorname{Dim}_{H} E_{\gamma}=1-H .
$$


3.2. Lower bound for $\operatorname{Dim}_{H} \mathcal{L}_{x}$

Recall $S_{n}$ from Section 2.1, and let us introduce the random variables

$$
Z_{n}^{x}=\frac{L^{x}\left(S_{n}\right)}{2^{n(1-H)}} \quad \text { and } \quad F_{N}^{x}=\sum_{n=1}^{N} Z_{n}^{x} .
$$

The random variables $\left(Z_{n}^{x}\right)_{n \geq-1}$ are positive, so $\left(F_{N}^{x}\right)_{N \geq 1}$ is non-decreasing. We denote by $F_{\infty}^{x}$ its limit, i.e. $F_{\infty}^{x}=\sum_{n=-1}^{\infty} Z_{n}^{x} \in$ $[0,+\infty]$.

Using (11), we have for all $n \geq 0$

$$
Z_{n}^{x} \stackrel{d}{=} Z_{0}^{2^{-n H} x}
$$

We note that similar random variables $Y_{n}^{x}=\frac{L^{2^{n} x}\left(S_{n}\right)}{2^{n(1-H)}}$ were introduced in Nourdin et al. (2018, Section 5.3). However, the fact that we are dealing with other space variables compared to Nourdin et al. (2018) induce several differences in our proofs. Although its statement is exactly the same than Nourdin et al. (2018, Lemma 5), the meaning and the context of our proof are different. This is why we provide all the details, for the convenience of the reader.

Our aim now is to link the random variable $Z_{n}^{x}$ to the microscopic Hausdorff dimension. To this end, let us introduce the random variables

$$
A_{n}:=\sup _{0 \leq t \leq 2^{n}} \sup _{0 \leq h \leq 2^{n-1}} \sup _{y \in \mathbb{R}} \frac{L^{y}([t, t+h])}{h^{1-H}\left(n-\log _{2} h\right)^{H}},
$$

where $\log _{2}$ stands for the binary logarithm (base 2). By (11), we have

$$
\begin{gathered}
A_{n}=\sup _{0 \leq t \leq 1} \sup _{0 \leq h \leq 1 / 2} \sup _{y \in \mathbb{R}} \frac{L^{y}\left(\left[2^{n} t, 2^{n}(t+h)\right]\right)}{\left(2^{n} h\right)^{1-H}\left(-\log _{2} h\right)^{H}} \\
\stackrel{d}{=} \sup _{0 \leq t \leq 1} \sup _{0 \leq h \leq 1 / 2} \sup _{y \in \mathbb{R}} \frac{L^{y}([t, t+h])}{h^{1-H}\left(-\log _{2} h\right)^{H}} .
\end{gathered}
$$

First, let us prove that $A_{n}$ is finite almost surely. We start by making use of a result of Xiao (1997, Theorem 1.2) that describes the scaling behavior of local times of Gaussian processes with stationary increments; in particular, this applies to the fractional Brownian motion and we have, with probability one:

$$
M:=\lim _{r \downarrow 0} \sup _{0 \leq t \leq 1} \sup _{0 \leq h \leq r} \sup _{y \in \mathbb{R}} \frac{L^{y}([t, t+h])}{h^{1-H}\left(-\log _{2} h\right)^{H}}<\infty .
$$

By the very definition of a limit, we deduce the existence of a (random) real number $0<r<1 / 2$ such that, almost surely,

$$
\sup _{0 \leq t \leq 1} \sup _{0 \leq h \leq r} \sup _{y \in \mathbb{R}} \frac{L^{y}([t, t+h])}{h^{1-H}\left(-\log _{2} h\right)^{H}} \leq 2 M .
$$

Now for $r \leq h \leq 1 / 2$, we have $h^{1-H}\left(-\log _{2} h\right)^{H} \geq r^{1-H}$ and $L^{y}([t, t+h]) \leq L^{y}([0,3 / 2])$ for all $0 \leq t \leq 1$ and $y \in \mathbb{R}$. Moreover by Xiao and Zhang (2002, Theorem 4.1), $B$ has a jointly continuous local time $(t, x) \mapsto L_{t}^{x}$ on $[0,3 / 2] \times \mathbb{R}$. Then, the (random) function $x \mapsto L_{t}^{x}$ is continuous on $\mathbb{R}$ and has a compact support (the occupation measure defined in Section 2.2 is compactly supported as $B([0,3 / 2])$ is compact). Hence, $\sup _{y \in \mathbb{R}} L^{y}([0,3 / 2])$ is finite and so one gets, almost surely,

$$
\begin{gathered}
\sup _{0 \leq t \leq 1} \sup _{r \leq h \leq 1 / 2} \sup _{y \in \mathbb{R}} \frac{L^{y}([t, t+h])}{h^{1-H}\left(-\log _{2} h\right)^{H}} \leq r^{H-1} \sup _{0 \leq t \leq 1} \sup _{r \leq h \leq 1 / 2} \sup _{y \in \mathbb{R}} L^{y}([t, t+h]) \\
\leq r^{H-1} \sup _{y \in \mathbb{R}} L^{y}([0,3 / 2])<\infty .
\end{gathered}
$$

Finally, by summing up (18) and (19), one has

$$
\mathbb{P}\left(\sup _{0 \leq t \leq 1} \sup _{0 \leq h \leq 1 / 2} \sup _{y \in \mathbb{R}} \frac{L^{y}([t, t+h])}{h^{1-H}\left(-\log _{2} h\right)^{H}}<\infty\right)=1 .
$$

Now for $K>0$ define the event

$$
\Omega_{K}:=\left\{\sup _{0 \leq t \leq 1} \sup _{0 \leq h \leq 1 / 2} \sup _{y \in \mathbb{R}} \frac{L^{y}([t, t+h])}{h^{1-H}\left(-\log _{2} h\right)^{H}} \leq K\right\} .
$$


Fix $x \in \mathbb{R}$ and consider the level set $\mathcal{L}_{x}$ defined by (2). By recalling Definition (7), we have: if $\left(I_{i}=\left[s_{i}, t_{i}\right]\right)_{i=1}^{m} \in \mathcal{I}_{n}\left(\mathcal{L}_{x}\right)$ is a cover minimizing $\widetilde{v}_{1-H, H}^{n}\left(\mathcal{L}_{x}\right)$ then,

$$
\widetilde{v}_{1-H, H}^{n}\left(\mathcal{L}_{x}\right)=\sum_{i=1}^{m}\left(\frac{\left|t_{i}-s_{i}\right|}{2^{n}}\right)^{1-H}\left|\log _{2} \frac{\left|t_{i}-s_{i}\right|}{2^{n}}\right|^{H} .
$$

Using (17) and a scaling argument with $t=\frac{s_{i}}{2^{n}}, h=\frac{t_{i}-s_{i}}{2^{n}}$, and $y=2^{-n H} x$, we deduce that

$$
\left(\frac{\left|t_{i}-s_{i}\right|}{2^{n}}\right)^{1-H}\left|\log _{2} \frac{\left|t_{i}-s_{i}\right|}{2^{n}}\right|^{H} \geq K^{-1} \frac{L^{x}\left(I_{i}\right)}{2^{n(1-H)}} \quad \text { on } \Omega_{K} .
$$

Back to (21), we have

$$
\widetilde{v}_{1-H, H}^{n}\left(\mathcal{L}_{x}\right) \geq K^{-1} \sum_{i=1}^{m} \frac{L^{x}\left(I_{i}\right)}{2^{n(1-H)}} \geq K^{-1} \frac{L^{x}\left(S_{n}\right)}{2^{n(1-H)}}=K^{-1} Z_{n}^{x}, \quad \text { on } \Omega_{K},
$$

where the last inequality holds because the local time $L^{x}$ increases only on the set $I_{i}$ (whose union covers $\mathcal{L}_{x} \bigcap S_{n}$ ). Finally, one gets

$$
\Omega_{K} \subset\left\{\forall x \in \mathbb{R}, \forall n \geq-1: \widetilde{v}_{1-H, H}^{n}\left(\mathcal{L}_{x}\right) \geq K^{-1} Z_{n}^{x}\right\} .
$$

Using (22) for the first inclusion and Lemma 4 for the second one, we can write

$$
\begin{aligned}
\Omega_{K} \cap\left\{\forall x \in \mathbb{R}, F_{\infty}^{x}=+\infty\right\} & \subset\left\{\forall x \in \mathbb{R}, \sum_{n \geq-1} \widetilde{v}_{1-H, H}^{n}\left(\mathcal{L}_{x}\right)=+\infty\right\} \\
& \subset\left\{\forall x \in \mathbb{R}, \operatorname{Dim}_{H} \mathcal{L}_{x} \geq 1-H\right\} .
\end{aligned}
$$

But by definition of $\Omega_{K}$ we have

$$
\mathbb{P}\left(\Omega_{K}\right) \underset{K \rightarrow+\infty}{\longrightarrow} \mathbb{P}\left(\sup _{0 \leq t \leq 1} \sup _{0 \leq h \leq 1 / 2} \sup _{y \in \mathbb{R}} \frac{L^{y}([t, t+h])}{h^{1-H}\left(-\log _{2} h\right)^{H}}<\infty\right)=1 .
$$

As a consequence, in order to conclude the proof of Theorem 2, it remains to check that $\mathbb{P}\left(\forall x \in \mathbb{R}, F_{\infty}^{x}=+\infty\right)=1$. Then, using (23), by letting $K \uparrow \infty$ an a.s. uniform lower bound of $\operatorname{Dim}_{H} \mathcal{L}_{x}$ is attained. The object of the next proposition is prove that $F_{\infty}^{x}=+\infty$ almost surely for all $x \in \mathbb{R}$.

Proposition 6. We have

$$
\mathbb{P}\left(\forall x \in \mathbb{R}, F_{\infty}^{x}=+\infty\right)=1
$$

Note that the following stronger statement of Proposition 6 was shown in Nourdin et al. (2018): for all $x \in \mathbb{R}$, $\mathbb{P}\left(F_{\infty}^{x}=+\infty\right)=1$. Our main contribution in the present note is precisely to prove the strongest version stated in Proposition 6.

\subsection{Proof of Proposition 6}

For every $a>0$, define

$$
\widetilde{Z}_{n}^{a}=\inf _{x \in[-a, a]} Z_{n}^{x} \text { and } \widetilde{F}_{\infty}^{a}=\sum_{n \geq 1} \widetilde{Z}_{n}^{a}
$$

Recalling (11), we get for all $n \geq 0$

$$
\widetilde{Z}_{n}^{a}=\inf _{x \in[-a, a]} Z_{n}^{x} \stackrel{d}{=} \inf _{x \in[-a, a]} Z_{0}^{2^{-n H} x}=\inf _{x \in\left[-2^{-n H} a, 2^{-n H} a\right]} Z_{0}^{x}=\widetilde{Z}_{0}^{2^{-n H} a} .
$$

In the three forthcoming lemmas, the following three facts are established:

(i) the existence of $\epsilon>0$ such that $\mathbb{P}\left(Z_{0}^{0}>4 \epsilon\right)>0$ (Lemma 7),

(ii) the existence of $a>0$ such that $\mathbb{P}\left(Z_{0}^{0}>4 \epsilon\right) \leq 2 \mathbb{P}\left(\widetilde{Z}_{0}^{a}>0\right)$ (Lemma 8),

(iii) that $\mathbb{P}\left(\widetilde{F}_{\infty}^{b}=\infty\right) \geq \mathbb{P}\left(\widetilde{Z}_{0}^{a}>0\right)$ for all $b>0$ (Lemma 9).

Combining the results obtained in (i) to (iii), we deduce that

$$
\mathbb{P}\left(\widetilde{F}_{\infty}^{b}=\infty\right)>0 \text { for all } b>0 .
$$


Set $\widehat{B}_{u}=u^{2 H} B_{1 / u}, u>0$. By the time inversion property of the fractional Brownian motion, $\widehat{B}$ is a fractional Brownian motion of Hurst index $H$ as well. We can write

$$
L^{x}\left(S_{n}\right)=\frac{1}{2 \pi} \int_{\mathbb{R}} d y e^{-i y x} \int_{2^{n-1}}^{2^{n}} d u e^{i y u^{2 H} \widehat{B}_{1 / u}} .
$$

As a result, we get that $x \mapsto L^{x}\left(S_{n}\right)$ is $\sigma\left\{\widehat{B}_{u}: u \leq 2^{-(n-1)}\right\}$-measurable, implying in turn that

$$
\sigma\left\{\widetilde{Z}_{n}^{b}: n \geq M\right\} \subset \sigma\left\{\widehat{B}_{u}: u \leq 2^{-(M-1)}\right\}
$$

for every $M \geq 1$. Consequently,

$$
\left\{\widetilde{F}_{\infty}^{b}=\infty\right\} \in \bigcap_{M \geq 1} \sigma\left\{\widehat{B}_{u}: u \leq 2^{-(M-1)}\right\} .
$$

Using (13), there exists a standard Brownian motion $\left(W_{u}\right)_{u \geq 0}$ defined on the same probability space such that

$$
\left\{\widetilde{F}_{\infty}^{b}=\infty\right\} \in \bigcap_{M \geq 1} \sigma\left\{W_{u}: u \leq 2^{-(M-1)}\right\} .
$$

By the Blumenthal's $0-1$ law, the probability $\mathbb{P}\left(\widetilde{F}_{\infty}^{b}=\infty\right)$ is either 0 or 1 . But by (28), this probability is strictly positive; hence we conclude that

$$
\mathbb{P}\left(\widetilde{F}_{\infty}^{b}=\infty\right)=1 \text { for all } b>0 .
$$

For every $b>0$, one has

$$
\begin{aligned}
& \mathbb{P}\left(\forall x \in[-b, b]: F_{\infty}^{x}=\infty\right)=\mathbb{P}\left(\inf _{x \in[-b, b]} F_{\infty}^{x}=\infty\right)=\mathbb{P}\left(\inf _{x \in[-b, b]} \sum_{N \geq 1} Z_{N}^{x}=\infty\right) \\
\geq & \mathbb{P}\left(\sum_{N \geq 1} \inf _{x \in[-b, b]} Z_{N}^{x}=\infty\right)=\mathbb{P}\left(\widetilde{F}_{\infty}^{b}=\infty\right)=1 .
\end{aligned}
$$

We finally conclude that

$$
\mathbb{P}\left(\forall x \in \mathbb{R}, F_{\infty}^{x}=\infty\right)=\lim _{b \rightarrow \infty} \mathbb{P}\left(\forall x \in[-b, b], F_{\infty}^{x}=\infty\right)=1,
$$

which is the desired conclusion of Proposition 6.

To conclude, it remains to state and prove the three lemmas mentioned in points (i) to (iii).

Lemma 7. There exists $\epsilon>0$ small enough such that $\mathbb{P}\left(Z_{0}^{0}>4 \epsilon\right)>0$.

Proof. Using that $L^{0}\left(\left[\frac{1}{2}, 1\right]\right)=\frac{1}{2 \pi} \int_{\mathbb{R}} d y \int_{\frac{1}{2}}^{1} d u e^{i y B_{u}}$, we have

$$
\mathbb{E}\left(L^{0}\left(\left[\frac{1}{2}, 1\right]\right)\right)=\frac{1}{2 \pi} \int_{\frac{1}{2}}^{1} u^{-H} d u \int_{\mathbb{R}} e^{-\frac{z^{2}}{2}} d z=\frac{1}{\sqrt{2 \pi}} \int_{\frac{1}{2}}^{1} u^{-H} d u>0 .
$$

As a result, $\mathbb{P}\left(Z_{0}^{0}>0\right)=\mathbb{P}\left(L^{0}\left(\left[\frac{1}{2}, 1\right]\right)>0\right)>0$, and the desired conclusion follows.

Lemma 8. For every $\epsilon>0$ small enough, there exists a real number $a>0$ such that

$$
0<\mathbb{P}\left(Z_{0}^{0}>4 \epsilon\right) \leq 2 \mathbb{P}\left(\widetilde{Z}_{0}^{a}>0\right) .
$$

Proof. Let $\beta<\frac{1}{2}\left(\frac{1}{H}-1\right), K=[-1,1]$ and $J=\left[\frac{1}{2}, 1\right]$. Set

$$
c=c(\omega):=\sup _{x \in K \backslash\{0\}} \frac{\left|L^{0}(J)(\omega)-L^{x}(J)(\omega)\right|}{|x|^{\beta}} .
$$

By Lemma 5, we have that $\mathbb{P}(c<\infty)=1$.

$$
\begin{gathered}
\text { Set } \eta_{\epsilon}=\eta_{\epsilon}(\omega):=\min \left\{\left(\frac{\epsilon}{c(\omega)}\right)^{1 / \beta}, 1\right\} \text {. As }\left[-\eta_{\epsilon}, \eta_{\epsilon}\right] \subset[-1,1] \text {, one has } \\
\forall|x| \leq \eta_{\epsilon}(\omega),\left|\left(L_{1}^{0}(\omega)-L_{1}^{x}(\omega)\right)-\left(L_{\frac{1}{2}}^{0}(\omega)-L_{\frac{1}{2}}^{x}(\omega)\right)\right| \leq \epsilon .
\end{gathered}
$$


By triangle inequality,

$$
\left|L_{1}^{x}-L_{\frac{1}{2}}^{x}\right| \geq\left|L_{1}^{0}-L_{\frac{1}{2}}^{0}\right|-\left|\left(L_{1}^{0}-L_{1}^{x}\right)-\left(L_{\frac{1}{2}}^{0}-L_{\frac{1}{2}}^{x}\right)\right| .
$$

Using (32) and (33), we have

$$
\left\{Z_{0}^{0}=L_{1}^{0}-L_{\frac{1}{2}}^{0}>4 \epsilon\right\} \subset\left\{\forall|x| \leq \eta_{\epsilon}(\omega),\left|L_{1}^{x}-L_{\frac{1}{2}}^{x}\right| \geq 3 \epsilon\right\} .
$$

But $\left\{\forall|x| \leq \eta_{\epsilon}(\omega),\left|L_{1}^{x}-L_{\frac{1}{2}}^{x}\right| \geq 3 \epsilon\right\}=\left\{\inf _{x \in\left[-\eta_{\epsilon}, \eta_{\epsilon}\right]}\left|L_{1}^{x}-L_{\frac{1}{2}}^{x}\right| \geq 3 \epsilon\right\}$. Recalling the definition of $\widetilde{Z}_{0}^{\eta_{\epsilon}}$, we deduce that

$$
\mathbb{P}\left(\widetilde{Z}_{0}^{\eta_{\epsilon}}>0\right) \geq \mathbb{P}\left(\widetilde{Z}_{0}^{\eta_{\epsilon}}>3 \epsilon\right) \geq \mathbb{P}\left(Z_{0}^{0}>4 \epsilon\right)>0 .
$$

Now for all $a>0$, we have

$$
\left\{\widetilde{Z}_{0}^{\eta_{\epsilon}}>0\right\} \subset\left\{\widetilde{Z}_{0}^{a}>0\right\} \cup\left\{\eta_{\epsilon} \leq a\right\} .
$$

Since $c<\infty$ a.s., one has that $\mathbb{P}(c \geq M) \rightarrow 0$ as $M \rightarrow \infty$. We can then choose $a>0$ small enough such that

$$
\mathbb{P}\left(\eta_{\epsilon} \leq a\right)=\mathbb{P}\left(c \geq \frac{\epsilon}{2 a \beta}\right) \leq \frac{1}{2} \mathbb{P}\left(Z_{0}^{0}>4 \epsilon\right) .
$$

Using (35), (36) and (37) we deduce that

$$
\mathbb{P}\left(Z_{0}^{0}>4 \epsilon\right) \leq \mathbb{P}\left(\widetilde{Z}_{0}^{\eta_{\epsilon}}>0\right) \leq \mathbb{P}\left(\widetilde{Z}_{0}^{a}>0\right)+\mathbb{P}\left(\eta_{\epsilon} \leq a\right) \leq \mathbb{P}\left(\widetilde{Z}_{0}^{a}>0\right)+\frac{1}{2} \mathbb{P}\left(Z_{0}^{0}>4 \epsilon\right) .
$$

Finally, this yields

$$
0<\mathbb{P}\left(Z_{0}^{0}>4 \epsilon\right) \leq 2 \mathbb{P}\left(\widetilde{Z}_{0}^{a}>0\right),
$$

which is the desired conclusion.

Lemma 9. For any $a, b>0$, we have

$$
\mathbb{P}\left(\widetilde{F}_{\infty}^{b}=\infty\right) \geq \mathbb{P}\left(\widetilde{Z}_{0}^{a}>0\right) .
$$

Proof. Fix $\gamma>0$ and $a, b>0$, consider the event $A_{\gamma, b}=\left\{\widetilde{F}_{\infty}^{b} \leq \gamma\right\}$. By Fubini's theorem,

$$
\gamma \geq \mathbb{E}\left(\mathbb{1}_{A_{\gamma, b}} \widetilde{F}_{\infty}^{b}\right)=\sum_{n \geq-1} \mathbb{E}\left(\mathbb{1}_{A_{\gamma, b}} \widetilde{Z}_{n}^{b}\right)=\sum_{n \geq-1} \int_{0}^{\infty} \mathbb{P}\left(A_{\gamma, b} \cap\left\{\widetilde{Z}_{n}^{b}>u\right\}\right) d u .
$$

Using $\mathbb{P}(A \cap B) \geq\left(\mathbb{P}(A)-\mathbb{P}\left(B^{c}\right)\right)_{+}$where $B^{c}$ denotes the complement of $B$, and recalling (27), we deduce that

$$
\gamma \geq \sum_{n \geq 0} \int_{0}^{\infty}\left(\mathbb{P}\left(A_{\gamma, b}\right)-\mathbb{P}\left(\widetilde{Z}_{n}^{b} \leq u\right)\right)_{+} d u=\sum_{n \geq 0} \int_{0}^{\infty}\left(\mathbb{P}\left(A_{\gamma, b}\right)-\mathbb{P}\left(\widetilde{Z}_{0}^{-n H_{b}} \leq u\right)\right)_{+} d u .
$$

There exists $M \geq 1$ such that $2^{-n H} b \leq a$ for all $n \geq M$. Then, for all $n \geq M$,

$$
\mathbb{P}\left(\widetilde{Z}_{0}^{2-n H} b \leq u\right) \leq \mathbb{P}\left(\widetilde{Z}_{0}^{a} \leq u\right)
$$

and

$$
\gamma \geq \sum_{n \geq M} \int_{0}^{\infty}\left(\mathbb{P}\left(A_{\gamma, b}\right)-\mathbb{P}\left(\widetilde{Z}_{0}^{a} \leq u\right)\right)_{+} d u .
$$

Since the summand does not depend on $n$ and the series is bounded by $\gamma$ and thus finite, one has necessarily

$$
\int_{0}^{\infty}\left(\mathbb{P}\left(A_{\gamma, b}\right)-\mathbb{P}\left(\widetilde{Z}_{0}^{a} \leq u\right)\right)_{+} d u=0
$$

Hence, for almost every $u \geq 0$ and every $\gamma \geq 0$,

$$
\mathbb{P}\left(\widetilde{F}_{\infty}^{b} \leq \gamma\right)=\mathbb{P}\left(A_{\gamma, b}\right) \leq \mathbb{P}\left(\widetilde{Z}_{0}^{a} \leq u\right) .
$$

We know that $\mathbb{P}\left(\widetilde{Z}_{0}^{a} \leq u\right)$ is increasing as a function of $u$. Hence, (38) is actually true for every $u \geq 0$ and $\gamma \geq 0$. Hence $\mathbb{P}\left(\widetilde{F}_{\infty}^{b}>n\right) \geq \mathbb{P}\left(\widetilde{Z}_{0}^{a}>\frac{1}{n}\right)$ for all $n \in \mathbb{N}$. One concludes that

$$
\mathbb{P}\left(\widetilde{F}_{\infty}^{b}=\infty\right) \geq \mathbb{P}\left(\widetilde{Z}_{0}^{a}>0\right) \text {. }
$$




\section{Acknowledgments}

This publication was supported by the FNR OPEN grantAPOGee at Luxembourg University. I thank my two advisors, Ivan Nourdin and Stéphane Seuret, for their guidance in the elaboration of this note. I am also grateful to an anonymous referee for the constructive comments and recommendations which helped me in correcting an error.

\section{References}

Barlow, M.T., Taylor, S.J., 1989. Fractional dimension of sets in discrete spaces. J. Phys. A 22 (13), 2621-2628, With a reply by J. Naudts.

Barlow, M.T., Taylor, S.J., 1992. Defining fractal subsets of $\mathbf{Z}^{d}$. Proc. Lond. Math. Soc. (3) 64 (1), $125-152$.

Baudoin, F., Nualart, D., 2003. Equivalence of Volterra processes. Stochastic Process. Appl. 107 (2), $327-350$.

Berman, S., Getoor, R., 1973. Local nondeterminism and local times of Gaussian processes. Indiana Univ. Math. 23 (1), 69-94.

Dozzi, M., 2003. Occupation density and sample path properties. In: Topics in Spatial Stochastic Processes. Springer, pp. 127-166.

Geman, D., Horowitz, J., 1980. Occupation densities. Ann. Probab. 1-67.

Khoshnevisan, D., Kim, K., Xiao, Y., et al., 2017. Intermittency and multifractality: A case study via parabolic stochastic pdes. Ann. Probab. 45 (6A), 3697-3751.

Khoshnevisan, D., Xiao, Y., 2017. On the macroscopic fractal geometry of some random sets. In: Stochastic Analysis and Related Topics. Springer, pp. 179-206.

Nourdin, I., 2012. Selected Aspects of Fractional Brownian Motion, Vol. 4. Springer.

Nourdin, I., Peccati, G., Seuret, S., 2018. Sojourn time dimensions of fractional Brownian motion. arXiv preprint arXiv:1809.00518.

Perkins, E., 1981. The exact Hausdorff measure of the level sets of Brownian motion. Z. Wahrscheinlichkeitstheor. Verwandte Geb. 58 (3), 373-388. Seuret, S., Yang, X., 2019. On sojourn of brownian motion inside moving boundaries. Stochastic Process. Appl. 129 (3), $978-994$.

Taylor, S.J., 1955. The $\alpha$-dimensional measure of the graph and set of zeros of a Brownian path. In: Mathematical Proceedings of the Cambridge Philosophical Society, Vol. 51. Cambridge University Press, pp. 265-274.

Xiao, Y., 1997. Hölder conditions for the local times and the Hausdorff measure of the level sets of Gaussian random fields. Probab. Theory Related Fields 109 (1), 129-157.

Xiao, Y., Zhang, T., 2002. Local times of fractional Brownian sheets. Probab. Theory Related Fields 124 (2), $204-226$. 